\section{COMUNICACIÓN PÚBLICA DE LA CIENCIA Y LA TECNOLOGÍA: UNA APROXIMACIÓN CRÍTICA A SU HISTORIA CONCEPTUAL}

\author{
Miguel Alcíbar \\ Universidad de Sevilla \\ jalcibar@us.es
}

\section{PUBLIC COMMUNICATION OF SCIENCE AND TECHNOLOGY: A CRITICAL APPROACH TO ITS CONCEPTUAL HISTORY}

Cómo citar este artículo/Citation: Alcíbar, M. (2015). "Comunicación pública de la ciencia y la tecnología: una aproximación crítica a su historia conceptual". Arbor, 191 (773): a242. doi: http://dx.doi.org/10.3989/arbor.2015.773n3012
Copyright: (c) 2015 CSIC. Este es un artículo de acceso abierto distribuido bajo los términos de la licencia Creative Commons Attribution-Non Commercial (by-nc) Spain 3.0.
RESUMEN: El artículo revisa críticamente la historia conceptual de la noción paraguas de comunicación pública de la ciencia y la tecnología (CPCT) y de los tres modelos básicos de comunicación surgidos a su amparo. Tres son los factores interdependientes que explican la emergencia de estos modelos: (1) cambios estructurales en la práctica científico-tecnológica y cambios concomitantes en los modos de comunicar la ciencia, (2) diversidad de actores sociales relacionados con la producción, gestión, comunicación y recepción de conocimientos científicos, y (3) desigual percepción que se tiene del público receptor.

PALABRAS CLAVE: comunicación pública de la ciencia y la tecnología; comprensión pública de la ciencia; ciencia y sociedad; ciencia en sociedad; modelo de déficit cognitivo; periodismo científico; divulgación de la ciencia; relaciones públicas en la ciencia.
ABSTRACT: The paper undertakes a critical review of the conceptual history of the umbrella notion of public communication of science and technology (PCST) and of the three basic models of communication it encompasses. Three closely intertwined factors are put forward explaining the emergence of these models: (1) structural changes in scientifictechnological practice and concomitant shifts in the modes of science communication; (2) the diversity of stakeholders in the production, management, communication and reception of scientific knowledge; and (3) differing audience perceptions.

KEYWORDS: public communication of science and technology; public understanding of science; science and society; science in society; cognitive deficit model; science journalism; popularization of science; public relations in science. 


\section{INTRODUCCIÓN: ACLARANDO CONCEPTOS}

En las últimas décadas, junto a la creciente influencia de la ciencia y la tecnología en el dominio económico, político y social, ha prosperado una suerte de visión ambivalente entre el público general, hasta el punto que hay autores que tildan la sociedad actual de esquizofrénica, esto es, una sociedad en la que coexisten dos tendencias opuestas: la tecno-optimista y la tecno-catastrofista (Felt, 2000; González García, López Cerezo y Luján López, 1996). De la ciencia se esperan soluciones efectivas a los graves problemas medioambientales, energéticos o de salud pública que encara la humanidad, pero también se temen las consecuencias negativas de sus aplicaciones tecnológicas. Se cree que esta ambivalencia puede socavar la legitimidad social y la autoridad cognitiva de las que ha gozado tradicionalmente la ciencia y, como consecuencia, esta puede verse cuestionada en la arena pública (Alcíbar, 2007). La comunicación pública de la ciencia y la tecnología (CPCT) desempeña un papel crucial en esta compleja realidad que involucra a científicos, tecnólogos, políticos, periodistas, empresarios, activistas y ciudadanos.

En este artículo entendemos la CPCT como una noción paraguas, es decir, una noción que cubre una miríada de actividades relacionadas con la ciencia y la tecnología en la sociedad ${ }^{1}$. Noticias de ciencia en un diario generalista o en el suplemento especializado de un periódico, libros escritos por científicos para el gran público, documentales de temática científica para la televisión, programas de radio dedicados a ciencia y tecnología, museos y exposiciones con contenidos científicos, Webs y Blogs de ciencia y tecnología, películas de ciencia ficción, etcétera, son algunos ejemplos relevantes de formatos, medios y mensajes en los que ciencia y tecnología se manifiestan fuera del núcleo cerrado de los especialistas. Dada esta diversidad, no es difícil concluir que uno de los obstáculos más serios al que se enfrentan los estudiosos de las relaciones ciencia-sociedad, es la dificultad para comprender de forma sistemática y coherente el amplio rango de actividades producidas por múltiples agentes sociales y destinadas a audiencias heterogéneas, tanto en niveles de alfabetización científico-tecnológica como en expectativas, intereses, creencias y valores. Por tanto, parece claro que la investigación y consolidación de la CPCT en el siglo XXI como disciplina requerirá de una mayor clarificación y profundización conceptual.

Para despejar posibles equívocos, es necesario hacer una distinción inicial entre ciencia y sociedad y ciencia en sociedad. Ambas expresiones sirven a dife- rentes instituciones públicas, como la Comisión Europea o la norteamericana National Science Foundation, para recomendar y/o gestionar políticas en materia de ciencia y tecnología ${ }^{2}$. La primera es una expresión clásica que alude a las relaciones que la ciencia establece con la sociedad en su conjunto. Se trata de un subproducto de la política científica que se instaura a partir del informe Science: the Endless Frontier, elaborado en 1945 por Vannevar Bush, ingeniero del MIT y asesor científico del presidente Roosevelt. El informe Bush aboga por una política de laissez faire, es decir, por una que separe la ciencia de la sociedad, para así preservar la autonomía de la primera de posibles injerencias políticas y/o sociales (Broks, 2006; Echeverría, 2003; Blanco e Iranzo, 2000). De esta manera, se asume que ambas son sistemas independientes y, por consiguiente, los esfuerzos se encaminan a tender puentes de entendimiento entre una y otra para que el ciudadano se identifique con las metas de la ciencia. Sin embargo, el supuesto hiato entre ciencia y sociedad no es algo esencial a la naturaleza de ambas, sino que más bien es un constructo social que atiende a específicos intereses políticos. La segunda expresión, más reciente, pretende destacar las interacciones dialógicas entre ciencia y sociedad, puesto que se entiende la ciencia como una institución social inserida en la sociedad y no independiente de esta, como sí parece sugerir la conjunción copulativa y en la locución clásica (European Commission, 2009; House of Lords, 2000). No obstante, hay expertos en CPCT que cuestionan la existencia de un cambio real de una perspectiva de déficit (ciencia y sociedad) a una de diálogo (ciencia en sociedad), ya que en las actividades basadas en un modelo dialógico persisten muchos de los problemas y ambigüedades del modelo de déficit cognitivo (Tinker, 2013; Trench, 2008). Así, modelos de comunicación que en teoría se sustentan en epistemologías y marcos de acción enfrentados, presentan en la práctica rasgos que pudieran revelar ideologías subyacentes similares, como es el caso de los modelos PAST y PEST (véase § 3).

Durante la historia conceptual de la CPCT, una rica terminología ha ido surgiendo como necesidad para denominar y, lo que es más importante, para dominar aspectos estratégicos de las relaciones ciencia-sociedad. Un mismo término puede emplearse con sentidos diferentes, según quién lo use, con qué propósitos y en qué contextos de aplicación. Esto se aprecia bien cuando se comparan dos grandes dominios sociales preocupados por la CPCT: el dominio político y el dominio académico. En el primero, instituciones de corte político que administran la ciencia elaboran informes 
prescriptivos para mejorar las relaciones ciencia-sociedad. Estas instituciones entienden la comunicación como una herramienta al servicio de los que toman decisiones. En el segundo dominio, expertos en estudios sociales de la ciencia elaboran textos académicos para examinar las relaciones ciencia-sociedad. Estos expertos entienden la comunicación como un objeto de investigación. Expresiones como comunicación científica (science communication), comprensión pública de la ciencia (public understanding of science), concienciación pública de la ciencia (public awareness of science) o compromiso público con la ciencia ( $p u$ blic engagement in science) se utilizan con diferentes matices en uno y otro dominio (Burns, O' Connor y Stocklmayer, 2003); lo mismo puede decirse de términos como divulgación de la ciencia (science popularization), periodismo científico (science journalism) y relaciones públicas (RR.PP) en la ciencia (public relations in science), que coexisten, se solapan y se han ido transformando durante la historia de la CPCT.

Nuestro objetivo principal es tratar de desbrozar la selva conceptual que rodea al fenómeno de la ciencia en sociedad. Para ello comenzamos situando en el centro del debate la noción de CPCT y los tres modelos de CPCT (con sus variantes léxicas) más citados en la literatura científica. Para entender cómo cada modelo se inspira en epistemologías distintas, es necesario considerar tres factores relacionados entre sí: (1) la existencia de cambios estructurales en la praxis científico-tecnológica, desde finales de la Segunda Guerra Mundial hasta nuestros días, así como los cambios concomitantes que han experimentado los modos de comunicar la ciencia, (2) la pluralidad de agentes sociales implicados en la producción, gestión, comunicación y recepción de conocimientos científicos, y (3) las desiguales percepciones que del público receptor tienen los distintos agentes sociales interesados en la CPCT.

La abundante literatura científica publicada en las últimas tres décadas hace que cualquier aproximación sea necesariamente parcial y selectiva. Este ensayo de revisión es, pues, un recorrido crítico de la historia conceptual de la CPCT.

\section{COMUNICACIÓN PÚBLICA DE LA CIENCIA Y LA TECNOLOGÍA, UNA NOCIÓN PARAGUAS}

Delimitar con precisión el concepto de CPCT es problemático (Trench y Bucchi, 2010). Su uso es coextensivo, haciéndose equivalente a otros términos igualmente paraguas, como el de comprensión pública de la ciencia o PUS, Public Understanding of Scien- ce (PUST, si se le añade Technology). A pesar de que CPCT y PUS no son sinónimos, en la práctica suelen intercambiarse. En un sentido lato, la CPCT describe el campo de comunicación entre científicos y no-científicos; los que investigan en él suelen adoptar una posición crítica respecto a los que utilizan estrategias comunicativas como recurso de poder. Se trata, pues, de un término que nace con la vocación de abarcar todas aquellas actividades con las que la cultura científica se incorpora a la cultura popular. Por su parte, la PUS es una expresión que surge a mediados de la década de 1980 con notable éxito y que gira en torno a la noción de alfabetización científica ${ }^{3}$. Los proponentes del llamado movimiento PUS esgrimen cuatro tipos de argumentos en su apoyo: (1) argumentos cientificos, al sugerir que la propia ciencia se beneficia de una mejor comprensión pública, tanto directamente, al despertar vocaciones científicas entre los jóvenes, como indirectamente, puesto que el apoyo político a la ciencia vendrá dado si los ciudadanos son más cultos y están más concienciados del valor intrínseco de la ciencia, (2) argumentos culturales, al enfatizar el papel de la ciencia al proporcionar significado y poner en valor la vida humana, (3) argumentos prácticos, al señalar las múltiples utilidades de comprender la ciencia -en el lugar de trabajo, en el hogar, en relación a la salud y seguridad personal, etc., y (4) argumentos políticos, al sugerir que la ciencia es crucial para la cohesión social y el buen funcionamiento de la democracia, puesto que su aportación es muy relevante para gestionar asuntos políticos de gran calado, como la política energética, la salud pública o la seguridad nacional (Durant, 2010).

Dentro del estudio de la PUS se observan dos perspectivas que difieren tanto en su epistemología como en sus ideas acerca del público, la comunicación y la ciencia. Por un lado, está la perspectiva tradicional, impulsada por aquellos que practican actividades encaminadas a mejorar la comprensión del público y comprometerlo con la ciencia (científicos, periodistas científicos, gestores de museos, etc.). Por otro lado, está la perspectiva crítica, promovida por investigadores sociales para explorar las interacciones públicociencia (Brossard y Lewenstein, 2010). Un ejemplo ilustrativo es el de los Blogs de ciencia (Luzón, 2013). Desde una perspectiva tradicional, el científico utiliza el Blog como una útil herramienta para, mediante la gestión de entradas (post), comunicar sus propias investigaciones a una audiencia heterogénea. Desde una perspectiva crítica, el científico social analiza los Blogs de ciencia y tecnología para averiguar cómo el gestor de estos Blogs emplea estrategias retóricas 
para influir en el público receptor. Cada perspectiva respalda modelos antagónicos de comunicación, ya que tanto los agentes que los emplean como los intereses a los que sirven son muy diferentes (véase $\S 3$ ).

Cabe señalar que, en general, bajo la rúbrica de CPCT se agrupan distintas prácticas que muestran aspectos comunes, pero también significativas diferencias. Dos de estas que, a menudo, se confunden son el periodismo científico y la divulgación de la ciencia. De acuerdo con Elías (2008), es necesario distinguirlas. El primero es un periodismo especializado que se caracteriza por situar socialmente los contenidos de naturaleza científica y/o tecnológica, contextualizando la fuente de información. Dicho de otra manera, busca la dimensión social de los descubrimientos científicos y de las innovaciones tecnológicas, de ahí que los asuntos que cubre estén muy apegados a la "rabiosa actualidad" (v. gr., nuevo acuerdo internacional para reducir las emisiones de $\mathrm{CO}_{2}$ para mitigar los efectos perniciosos del cambio climático). Suele practicarlo el periodista y su público diana es muy heterogéneo. La segunda, la divulgación de la ciencia, se centra en hacer accesibles contenidos estrictamente científicotécnicos, lo que hace que sus temas sean atemporales (v. gr., nucleosíntesis estelar). Habitualmente la practica el científico que posee ciertas habilidades comunicativas y su potencial audiencia suele ser más concreta, por lo general, personas previamente interesadas, estudiantes y otros científicos.

Además, en las tres últimas décadas ha arraigado entre los comunicólogos de tendencia crítica una expresión relacionada con el creciente fenómeno de la medialización de la ciencia (medialization of science): las relaciones públicas en la ciencia. La ciencia depende cada vez más de recursos humanos y financieros escasos, así como de su aceptación social (opinión pública, políticos, sponsors). Estas restricciones explican, en gran medida, que en la actualidad la actividad de muchos científicos esté muy orientada hacia los medios de comunicación (Peters, 2012; Rödder, 2009; Weingart, 1998). En determinadas áreas de investigación sensibles socialmente es habitual que los científicos (a título personal o gracias a knowledge brokers), adopten herramientas propias de las relaciones públicas y la comunicación corporativa como parte de sus estrategias comunicativas. De esta manera pueden hacerse visibles en los foros públicos y proyectar una imagen positiva de sí mismos y de sus actividades. A veces, este afán de visibilidad pública hace que el científico se arrogue actitudes sensacionalistas o exageradas que suele censurar cuando las exhibe el pe- riodista. En su ya clásico e interesante libro La ciencia en el escaparate, Dorothy Nelkin (1990) relata cómo un científico, ávido por atraer el interés mediático, sobreestimó y exageró los beneficios terapéuticos de haber aislado el gen responsable del cáncer de colon, declarando a un reportero del New York Times que con ello podía evitarse la muerte. Otro buen ejemplo de orientación de la ciencia hacia los medios es el Proyecto Genoma Humano, puesto que la presentación pública de la secuencia completa del genoma fue todo un alarde de maquinaria publicitaria (Rödder, 2009; Nerlich, Dingwall y Clarke, 2002). Estos y otros ejemplos demuestran que los científicos son capaces de explotar los medios como un recurso político, sobre todo cuando los asuntos que se manejan están sujetos a competición o controversia (Elliott, 2012).

Así pues, la CPCT es una actividad compleja y muy dependiente del contexto. Aunque engloba una gran variedad de contenidos, estrategias, formatos, valores, propósitos y funciones, se la ha querido reducir a un mero mecanismo de transmisión lineal de conocimientos genuinos, desde un dominio de autoridad (el científico), que selecciona y difunde contenidos estratégicos, hasta otro lego (el público), que recibe la información científica de manera indiferenciada y pasiva. Este enfoque dominante sirve al científico como recurso de acción política al atribuirle la potestad de sancionar qué es "simplificación adecuada" y qué "contaminación" del discurso científico original, es decir, qué es buena y qué es mala divulgación (Hilgartner, 1990).

Las tres formas de CPCT descritas más arriba no son compartimentos estancos, sino que tienen zonas de solapamiento. Por ejemplo, en el periodismo científico hay contenidos divulgativos y en las actividades de relaciones públicas se adoptan tácticas propias del periodismo y de la divulgación. Tampoco quedan definidas siempre las intenciones que animan a quienes divulgan, promocionan, producen o informan de conocimientos científicos. Estas "zonas de indefinición" han llevado a ciertos autores a proponer modelos contextuales que conciben la CPCT como un continuum (Bucchi, 1998; Hilgartner, 1990).

\section{MODELOS DE COMUNICACIÓN PÚBLICA DE LA CIENCIA Y LA TECNOLOGÍA}

Sarah Tinker (2013) realiza una revisión crítica de lo que ella denomina modelos de popularización de la ciencia. En nuestra opinión, se está refiriendo a modelos de CPCT y no tanto a modelos circunscritos a un solo aspecto de la CPCT, como es el caso de la popularización científica. Los tres modelos que des- 
cribe Tinker (PAST, PEST y CUSP), concuerdan con los principales modelos que durante décadas vienen discutiendo los expertos en CPCT. Abarcan un espectro continuo que va de los modelos unidireccionales, gestionados por fuentes de autoridad científica, a los interactivos y críticos, implementados por expertos en comunicación y que pretenden aglutinar todos los elementos que participan de la ciencia en sociedad. En la práctica, estos modelos no funcionan como diseños puros de comunicación, sino que se imbrican y fertilizan entre sí. Cada uno de ellos enfatiza algún aspecto del proceso comunicativo.

\subsection{Modelo de apreciación pública de la ciencia y la tecnología (PAST, Public Appreciation of Science and Technology)}

Es el modelo más elemental y más aplicado porque el flujo de información va de la ciencia, el diseminador activo y quien controla el significado de "lo científico" y "lo no-científico", al público, un mero depósito pasivo de información. Por definición, el público es ignorante y refractario y por eso hay que educarlo y/o adoctrinarlo por medio de información persuasiva. Para Tinker, PAST es un término más adecuado que el clásico PUS porque en realidad no existe una "comprensión" sino una "apreciación" de la ciencia (véase § 2). En efecto, para los proponentes de este modelo es prioritario que el público aprecie el valor intrínseco de la ciencia, aunque constaten una y otra vez (mediante encuestas periódicas de percepción) que esta modo unidireccional de comunicar la ciencia no contribuye de manera significativa a incrementar la cultura científica de la población. EI PAST atiende a otras denominaciones, tales como modelo de déficit cognitivo o de alfabetización científica (Brossard y Lewenstein, 2010; Väliverronen, 1993), modelo de diseminación/difusión (Horst, 2008), enfoque dominante (Myers, 2003; Hilgartner, 1990) o enfoque canónico de popularización de la ciencia (Grundmann y Cavaillé, 2000; Bucchi, 1998).

El modelo PAST es problemático por varias razones. En primer lugar, tiende a considerar que la ciencia es una "caja negra", una especie de reservorio de hechos que tienen su correspondencia exacta con fragmentos del mundo natural, con lo que ignora que el conocimiento científico es un constructo social (Woolgar, 1991). En segundo lugar, asume que el científico posee autoridad cognitiva per se, con lo que olvida que "especialista" y "no especialista" son conceptos relativos y que el "especialista" solo es experto en el restringido campo de su especialidad (Fehér, 1990). En tercer lugar, considera que el déficit cognitivo se ciñe en exclusiva al "no científico". Si el público no reconoce los hechos científicos (que son como son), tal falla se imputa a deficiencias en los mediadores al transmitir la información o a creencias irracionales del público, o a ambas. En cuarto lugar, se basa en un generalizado, aunque obsoleto, lineal y demasiado simplista modelo de comunicación (Hansen, 2009). No fomenta la participación pública y refleja únicamente las expectativas e intereses de una élite cientificista y tecnocrática. En efecto, este modelo tiene como piedras angulares una ideología cientificista y una concepción positivista de la alfabetización científica, con lo cual está centrado en la ciencia, es paternalista y pedagógico (Väliverronen, 1993). En quinto lugar, no funciona realmente puesto que no cumple con las expectativas de quienes lo impulsan y defienden. Tras décadas de denodados esfuerzos por sensibilizar al público mediante campañas informativas y programas educativos informales, no se ha logrado incrementar la comprensión científica de este (Logan, 2001; Miller, 2001). En sexto y último lugar, no es necesario. En contra de la opinión de algunos científicos y gestores que aseveran que los ciudadanos tienen una fuerte resistencia a apoyar la ciencia porque han perdido la fe en ella, los estudios sobre percepción social de la ciencia y la tecnología muestran que, en realidad, no existe una falta de interés, respeto o confianza hacia la ciencia en general, sobre todo entre las audiencias con mayor nivel cultural (FECYT, 2015; Miller, 2004; Felt, 2000; Evans y Durant, 1995; Durant, Evans y Thomas, 1989).

\subsection{Modelo de compromiso público con la ciencia y la tecnología (PEST, Public Engagement with Science and Technology)}

EI PEST es un modelo que trasciende la noción de déficit porque concibe la comunicación como un flujo bidireccional entre ciencia y público. En los últimos años se ha convertido en un paradigma esencial para algunas instituciones de investigación dentro y fuera de Europa, cuya voluntad es obtener el compromiso del público mediante el fomento de actividades científicas (outreach activities) (Bucchi, 2013, p. 905). La diferencia con el modelo anterior es que enfatiza la necesidad de establecer mecanismos que favorezcan el diálogo entre ciencia y sociedad, por ello también se lo conoce como modelo de diálogo (Brossard y Lewenstein, 2010; Trench, 2008; Miller, 2001).

Sin embargo, como bien argumenta Tinker, aunque el PEST mejora el PAST también presenta problemas. En primer lugar, sigue separando ciencia y sociedad, 
incluso cuando pretende establecer un diálogo entre ambas. Según Broks (2006), lo que ocurre es que "el nuevo lenguaje de diálogo oculta el viejo objetivo de las relaciones públicas", propio del PAST. En segundo lugar, sigue manteniendo el centro de gravedad en la ciencia: la ciencia es una entidad fija, sin fisuras, hacia la que el público debe moverse para comprometerse con ella. A pesar de que el PEST asume que los miembros del público son agentes activos que se empoderan en sus interacciones con la ciencia, todavía enfatiza que el cambio debe darse en el público. En tercer lugar, al sugerir que las preocupaciones del público se deben a falsas apreciaciones de los hechos científicos más que a reticencias de fondo, sus postulantes asumen la visión de la ciencia como una "caja negra". Así, por ejemplo, los impulsores del PEST abogarían por establecer procesos de diálogo entre todos los agentes sociales implicados en una controversia tecnocientífica con derivaciones sociales, como es el caso de la instalación de antenas de telefonía móvil en viviendas, pero evaluarían los temores de los detractores más como fruto de la ignorancia que como una oposición basada en preocupaciones legítimas, sean de salud pública, éticas o políticas.

Algunos autores, solo ven posible un verdadero diálogo simétrico entre lego y experto gracias a la irrupción de los nuevos medios sociales, puesto que facilitan la formación de comunidades online y la participación directa del ciudadano en la política científica y en los debates científicos con implicaciones éticas, legales y sociales (Peters, 2013).

\subsection{Modelo de comprensión crítica de la ciencia en pú- blico (CUSP, Critical Understanding of Science in Public)}

EI CUSP supera a los dos anteriores modelos en considerar críticamente todos los aspectos que intervienen en las interacciones ciencia-sociedad. En este sentido, el CUSP reconoce que la comunicación no se puede reducir a un simple proceso lineal de difusión de información y, por consiguiente, subraya su carácter multidimensional y contextual. El problema ya no es identificar los motivos por los que el público ignora la ciencia sino que más bien se trata de establecer cauces para que este pueda alcanzar una comprensión crítica del fenómeno científico y, por tanto, pueda cuestionar y responder a los pros y contras que suscita la tecnociencia (Horst, 2008). Por tanto, como apuntan Collins y Pinch (1996), el público debe aprender más acerca de la ciencia (institución social) que de ciencia (contenidos). Este modelo también se conoce como modelo de deliberación (Horst y Michael, 2011) y se le relaciona con el modelo democrático (Durant, 1999) y con el modelo contextual (Gross, 1994).

Hay dos diferencias cardinales entre el CUSP y los dos modelos anteriores. La primera estriba en la distinta base epistemológica; mientras el PAST y el PEST se fundamentan en principios de corte positivista, el CUSP tiene una base marcadamente constructivista. Es por esta razón que este último se centra sobre todo en la producción de sentido. Dicho de otra manera: los defensores de este modelo no atienden tanto a la exactitud con la que se trasmiten los hechos científicos, desde una fuente experta a un sumidero lego, como a que los hechos emergen y se mantienen en los foros públicos gracias a complejas interacciones entre las intenciones y necesidades de productores y consumidores de textos, en contextos sociales y culturales dados. El conocimiento y su difusión siguen importando pero se pone más el énfasis en cómo se usa socialmente ese conocimiento. La segunda diferencia atañe al origen de estos modelos. Mientras que el PAST y el PEST son implementados por instituciones científicas o por organismos públicos dedicados a la política científica, el CUSP nace de la reflexión crítica propia del mundo académico.

EI CUSP ofrece cuatro ventajas sobre los modelos anteriores, a saber: (1) tiene un marcado carácter relacional y subraya que la ciencia es una parte interactiva de la sociedad. Reconoce, por tanto, que la ciencia no está aislada de otras instituciones sociales, (2) considera que las formas de conocimiento científicas y las no-científicas tienen valor. Esto implica que la participación de todos los agentes sociales involucrados en los debates públicos sobre asuntos tecnocientíficos es necesaria para alcanzar el consenso, (3) propone una doble obligación para la CPCT. Por una parte, la de informar y educar al público y, por otra, la de explorar y criticar a la ciencia, como institución social que es. Este papel inquisitivo y crítico está ausente en los modelos PAST y PEST y, además, es ineludible porque siempre se da cierta apropiación social de conocimiento científico, es decir, de utilización de este en determinados contextos extra-científicos (cf. Sørensen, Aune y Hatling, 2000), y (4) asume que hay una imagen pública de la ciencia ambivalente, resultado del entusiasmo que generan los logros de la investigación científica (sobre todo en el área de la biomedicina) y de la suspicacia que provocan sus aplicaciones y que se cree pueden socavar la calidad de la vida humana (y a menudo de la no humana) (véase § 4.3). 


\section{FACTORES EXPLICATIVOS DE LA VARIEDAD DE MODELOS EN LA CPCT}

Una vez delimitada la noción de CPCT y sus distintos enfoques, es imprescindible plantear qué factores explican por qué, cuándo, dónde y cómo surgen estos modelos y su terminología asociada. La terminología es amplia, ya que, como se ha visto, distintos autores etiquetan de manera disímil conceptos que son equivalentes, como es el caso del modelo PAST y el de déficit cognitivo. A continuación se describen los tres factores que, a nuestro entender y de modo sinérgico, explican la emergencia y proliferación terminológica en la CPCT.

\subsection{Cambios estructurales en la práctica científica y en los modos de comunicación de la ciencia}

Si la revolución científica que se produce en Europa en los siglos XVI y XVII comporta cambios en la estructura del conocimiento, la actual revolución tecno-científica, cuyo origen puede situarse en EE.UU en la época de la Segunda Guerra Mundial, conlleva cambios en la estructura de la práctica científico-tecnológica (Echeverría, 2003). En efecto, en el periodo de postguerra empiezan a darse profundos cambios en el sistema ciencia-tecnología norteamericano que contribuyen al surgimiento de la tecnociencia. Entre estos profundos cambios cabe señalar el incremento de la burocratización, politización y mercantilización de la ciencia. Lo que aquí nos interesa, sin embargo, es que todas estas transformaciones implican cambios concomitantes en la manera que los científicos tienen de interactuar con otros agentes sociales y en los modos de comunicar la ciencia y la tecnología.

El Informe Bush, como ya se apuntó, perfila las bases de la política científica de EE.UU para tiempos de paz y determina que el gobierno facilite las condiciones necesarias para que los científicos trabajen sin problemas financieros y sin injerencias externas, a cambio de que proporcionen conocimiento científico fiable y beneficioso para el progreso económico y social de la nación. Es lo que se conoce como contrato social en pro de la ciencia o antiguo contrato entre ciencia y sociedad que promueve, desarrolla y transforma el contexto en el que los científicos investigan y los tecnólogos innovan (Meyer, 2006; Echeverría, 2003). Muchos científicos e ingenieros se incorporan al núcleo duro del poder político, lo cual implica que la actividad científica se impregne de valores políticos y jurídicos.
Las políticas neoliberales aplicadas en los años ochenta por Ronald Reagan, en Estados Unidos, y Margaret Thatcher, en Gran Bretaña, empiezan a resquebrajar este antiguo contrato social. El impulso neoliberal conlleva la desregulación de los mercados, la mercantilización de la investigación tecnocientífica, la privatización del conocimiento y la pérdida de autonomía de los científicos. Se implanta así un nuevo contrato entre ciencia y sociedad que favorece el rápido incremento de la financiación privada en I+D, gracias a la liberalización de las patentes (la conocida Acta Bayh-Dole) y una nueva política fiscal que permite desgravar un $25 \%$ de las inversiones privadas en $I+D$ (Echeverría, 2003). La tecnociencia ni es autónoma ni es neutral.

A partir de un estudio a gran escala sobre la ciencia publicada entre 1945 y 1990 por la prensa británica, Bauer y Gregory (2008) identifican claramente dos periodos de prominencia mediática de la ciencia y la tecnología: uno entre 1956 y 1966, con un pico en $1960 / 62$, y otro después de 1992. Además, señalan que, a partir de mediados de la década de 1970, se dan unos años de transición en los que el declive en la cobertura de los años sesenta llega a su cota más baja para comenzar luego a crecer hasta llegar, en la década de 1990, a su punto más álgido. La primera expansión coincide con el auge de la astronomía, el comienzo de la carrera espacial y la energía nuclear, actividades todas financiadas por el gobierno mediante programas nacionales. En la segunda mitad de los sesenta, tras el fracaso norteamericano en la guerra del Vietnam y la crisis energética de 1973, surgen robustos movimientos sociales y la desconfianza hacia la ciencia crece. Esto tiene un evidente efecto negativo en el presupuesto público dedicado a la investigación científica. El interés de la prensa por el espacio y la energía nuclear se va sustituyendo, primero, por temas medioambientales, luego, por la computación y, más tarde, ya en plena década de 1990, por la biotecnología. Este cambio en la cobertura periodística de las ciencias físicas a las biomédicas, descrito como medicalización de las noticias científicas (Bauer, 1998), coincide con la tendencia a privatizar la ciencia, sobre todo en áreas con implicaciones sociales como la biotecnología y la biomedicina. El incremento de la financiación privada propicia la irrupción en la ciencia de profesionales de las relaciones públicas, más interesados en promocionar la imagen corporativa de quien detenta y gestiona el conocimiento que en trasladar al ciudadano las incertidumbres que impregnan toda empresa tecnocientífica (Bauer y Gregory, 2008). 
Lo que Bauer y Gregory describen son cambios significativos en el modo de comunicar la ciencia. De una lógica periodística (que se adapta al clima social de cada momento), se pasa en los años 90 a una lógica característica de las relaciones públicas. Se opera, en definitiva, una drástica permuta en el control del flujo de información: si en la década de 1950 el control es básicamente periodístico, en la actualidad es institucional. Estos autores etiquetan este cambio como PUS Inc.

La Tabla I sintetiza los cambios más notables producidos en cada periodo, tanto en las prácticas tecnocientíficas y los contenidos mediáticos sobre ciencia y tecnología como en los modos de comunicación.

Sin embargo, estos cambios en la práctica científica no conllevan, como sería deseable, un ajuste crítico en las rutinas profesionales del periodismo científico actual. Así, el viejo régimen (Bauer y Gregory, 2008) o convención tradicional (Meyer, 2006) del periodismo científico, un evidente subproducto del antiguo contrato social con la ciencia, no ha devenido en un necesario periodismo científico crítico. La irrupción en los años ochenta de la empresa privada y la consiguiente mercantilización de la ciencia académica im- plica el predominio de criterios económicos, aunque no su absoluta prevalencia. Esto puede tener un impacto indeseable sobre métodos y resultados en la investigación, conducir a un mayor nivel de secretismo, disminuir los proyectos científicos de interés público y aquellos que se consideran poco rentables, desde una perspectiva puramente economicista, o estimular las malas conductas entre los científicos (Radder, 2010). Así, hoy día, impera lo que Bauer denomina "para-periodismo", un periodismo acrítico que atiende a una lógica corporativa en detrimento de una periodística (Bauer y Gregory, 2008; Meyer, 2006).

Adaptando las ideas de Bauer y Gregory (2008), la Tabla II resume y confronta los rasgos propios de la comunicación basada en una lógica periodística (periodismo científico) y en una lógica de relaciones públicas (PUS Inc.).

En resumen, los cambios en la práctica científica han tenido su correlato en los modos de comunicar la ciencia, tanto en contenidos como en estrategias. Estos cambios han contribuido a configurar un complejo mapa conceptual de la CPCT. Si en el primer periodo (1950-1965) los logros de la ciencia son accesibles gracias a entusiastas periodistas y científicos, con un

Tabla I. Resumen de los cambios en los modos de comunicación de la ciencia (Adaptado de Bauer y Gregory, 2008)

\begin{tabular}{|c|c|c|c|}
\hline PERIODO & PRÁCTICAS TECNOCIENTÍFICAS & $\begin{array}{c}\text { CONTENIDO DE CIENCIA Y } \\
\text { TECNOLOGÍA }\end{array}$ & MODO DE COMUNICACIÓN \\
\hline $1940-1965$ & $\begin{array}{l}\text { Emerge la macrociencia, tenien- } \\
\text { do la investigación básica un } \\
\text { destacado papel como motor de } \\
\text { ésta (Física, Química) } \\
\text { Financiación gubernamental }\end{array}$ & $\begin{array}{l}\text { Ciencias físicas } \\
\text { Energía nuclear } \\
\text { Carrera espacial entre EE.UU } \\
\text { y URSS } \\
\text { Proyectos con financiación } \\
\text { pública }\end{array}$ & $\begin{array}{l}\text { Convención tradicional del periodis- } \\
\text { mo científico } \\
\text { La comunicación social que realizan } \\
\text { los científicos tiene un marcado ob- } \\
\text { jetivo educativo y promocional }\end{array}$ \\
\hline 1966-1976 & $\begin{array}{l}\text { Década de crisis y estancamien- } \\
\text { to, provocada por el fracaso de } \\
\text { EE.UU en Vietnam y la crisis } \\
\text { energética de } 1973 \\
\text { Surgimiento de movimientos de } \\
\text { protesta contra la macrociencia } \\
\text { militarizada }\end{array}$ & $\begin{array}{l}\text { Ambientalismo } \\
\text { Protestas anti-nucleares } \\
\text { Guerra contra el cáncer } \\
\text { Límites en el crecimiento po- } \\
\text { blacional (Club de Roma) }\end{array}$ & $\begin{array}{l}\text { Periodo de transición } \\
\text { Periodismo escéptico y crítico en la } \\
\text { línea de los movimientos sociales de } \\
\text { protesta }\end{array}$ \\
\hline 1977-actualidad & $\begin{array}{l}\text { Surge la tecnociencia propia- } \\
\text { mente dicha, impulsada por al- } \\
\text { gunas grandes empresas, más } \\
\text { que por el Estado } \\
\text { La tecnociencia se centra en las } \\
\text { nuevas tecnologías } \\
\text { Financiación privada global }\end{array}$ & $\begin{array}{l}\text { "Medicalización" de las noti- } \\
\text { cias científicas: ciencias bio- } \\
\text { médicas, biotecnología }\end{array}$ & $\begin{array}{l}\text { Nuevo régimen del movimiento PUS } \\
\text { (PUS Inc.) } \\
\text { Promoción de la imagen pública de } \\
\text { la ciencia } \\
\text { Estrategias de RR.PP y comunicación } \\
\text { corporativa } \\
\text { Para-periodismo; eventos mediáti- } \\
\text { cos (v. gr., conferencias de prensa) }\end{array}$ \\
\hline
\end{tabular}


Tabla II. Rasgos enfrentados de las dos lógicas en la comunicación de la ciencia (Adaptado de Bauer y Gregory, 2008)

\begin{tabular}{ll}
\hline \multicolumn{1}{c}{ LÓGICA PERIODÍSTICA } & \multicolumn{1}{c}{ LÓGICA PUBLICITARIA } \\
\hline Depende del clima social del momento (celebratorio, escéptico) & Depende de los intereses corporativos a los que sirve (celebratorio) \\
\hline Creación de relatos noticiosos & Creación de eventos mediáticos \\
\hline Debate, polémica, controversia & Conferencia de prensa, comunicado de prensa \\
\hline Científico visible en los medios & Científico-estrella puesto por profesionales de las relaciones públicas \\
\hline Científico como héroe o villano & Imagen positiva de la corporación \\
\hline Intercambio de conocimiento & Creación de imagen pública \\
\hline Público como ciudadano & Público como consumidor y accionista \\
\hline Foro público (ágora) & Feria de la ciencia (exhibiciones) \\
\hline
\end{tabular}

fuerte sentido didáctico de su profesión, a partir de la década de 1980 son los profesionales de las relaciones públicas los que controlan el flujo de información científica. Esto explica la ubicuidad de los modelos PAST y PEST y la presencia residual del modelo CUSP.

\subsection{Pluralidad de actores implicados en la producción, gestión, comunicación y recepción de conocimientos científicos}

La CPCT y la PUS son términos que adquieren sentidos diferentes, según quién los emplee (Weigold, 2001). Por una parte, científicos, periodistas, gestores de museos de ciencia, miembros de sociedades e instituciones científicas, administradores de la política científica y profesionales de las relaciones públicas en centros de investigación y revistas especializadas los utilizan para subrayar la necesidad de que el público se involucre con una amplia categoría de actividades y se alinee con las prioridades y preocupaciones de los científicos. Estos son los que Tinker (2013) llama impulsores de la ciencia (science boosters). Una de las consecuencias del movimiento PUS (PUS Inc.) es "la institucionalización de la comunicación de la ciencia como un acreditado componente de la educación formal" (Bauer y Gregory, 2008). Se piensa que cuantos más conocimientos posea el ciudadano mayor será su compromiso con la ciencia; por tanto, "comprensión de la ciencia" se equipara a "apreciación de la ciencia" (Tinker, 2013; Gregory y Miller, 1998; Wynne, 1995; Lewenstein, 1992). La vocación pedagógica impregna la terminología. Así, muchas instituciones científicas organizan cursos de formación dirigidos a estudiantes de ciencias (no periodistas) para que se incorporen a agencias de relaciones públicas cuya misión es servir a los intereses corporativos y comerciales de las organizaciones para las que trabajan. Por otra parte, expertos en comunicación conciben la CPCT y la PUS como fructíferos campos de investigación en la que se debe combinar la apreciación con la evaluación argumentada de la ciencia. A unos les preocupa teorizar sobre la forma más eficiente de comunicar y conectar con el público (modelo PAST y subyacentemente el PEST). A otros les interesa establecer vías de diálogo más realistas y críticas que pongan en valor el papel del ciudadano en la toma de decisiones sobre asuntos tecnocientíficos complejos (modelo CUSP). Estos son los llamados críticos de la ciencia (science critics) (Tinker, 2013).

El cambio del antiguo al nuevo contrato social (véase $§ 4.1$ ), apunta a un desplazamiento de los tradicionales e institucionales lugares (gobierno, industria, universidad) en los que se debaten, formulan y negocian los problemas, al "ágora", el espacio público donde las grandes cuestiones tecnocientíficas se encuadran y definen, y sus "soluciones" se negocian; esto es, donde "la ciencia se encuentra con el público" y el público le "responde" (Gibbons, 1999). Los medios sociales y las nuevas TIC desempeñan un papel cada vez más activo en el ágora, lo que trae consigo nuevos actores que se involucran en la producción, gestión, comunicación y recepción de conocimientos científicos.

La manera de encuadrar los problemas, definirlos y proponer sus posibles soluciones nos revela esta pluralidad de actores sociales. Este proceso se ha dado en llamar framing y a los encuadres que se utilizan, frames. Se piensa que el público general usa los frames como esquemas interpretativos para dotar de sentido determinados asuntos y poder así discutir sobre ellos. También los usan los periodistas para condensar los aspectos más relevantes de un suceso complejo y hacerlo accesible a amplias audiencias, los responsables políticos para definir opciones políticas y tomar decisiones, y los científicos (y de forma análoga los profesionales de las relaciones públicas que trabajan para 
instituciones científicas) para encuadrar públicamente los resultados de sus investigaciones (Nisbet, 2010).

En síntesis, los cambios operados en la ciencia de post-guerra implican que los proyectos de investigación sean cada vez más complejos, controvertidos y sujetos al escrutinio público. Se favorece así la proliferación de nuevos agentes activos. A científicos y periodistas hay que sumar responsables políticos, empresarios, legisladores, activistas, profesionales de las relaciones públicas, entre otros, que compiten por configurar nuevos modos de comunicar la ciencia, introducir conceptos o matizar otros ya existentes. Esto les permite diseñar un amplio conjunto de actividades cuyos propósitos específicos van más allá del mero intento de entusiasmar al público con la ciencia.

\subsection{Desigual percepción que se tiene del público receptor}

Para muchos estudiosos el público es el componente menos conocido y uno de los más problemáticos en la CPCT (Felt, 2000; Fehér, 1990). Fue a partir de mediados del siglo XVIII, en el tránsito de la etapa amateur a la moderna en el proceso de institucionalización social de la ciencia, cuando científicos y filósofos asignan al público un papel subordinado y pasivo en relación a la autoridad de los expertos (Woolgar, 1991). Este nuevo papel está relacionado con el creciente volumen de conocimientos y la segmentación de la ciencia en especialidades autónomas. El público es una masa indiferenciada de consumidores pasivos de descubrimientos científicos e innovaciones tecnológicas. Se establece, por tanto, un modelo lineal de comunicación entre la autoridad científica y el público lego. La información invariablemente fluye en un único y simple sentido: de productores a consumidores, forzando a quienes median (sobre todo, periodistas y knowledge brokers) a asumir el papel de meros amplificadores y traductores. Además, esta frontera retórica entre experto y lego conlleva la estricta segregación del conocimiento científico genuino del divulgativo, quedando este último relegado a un estatus inferior (Hilgartner, 1990).

En el fundamento del modelo de déficit cognitivo está la presunción de que la falta de apoyo simbólico y material que se cree manifiesta el público hacia la ciencia se debe a sus carencias educativas. De facto el público es ignorante y, por consiguiente, no está cualificado para deliberar y decidir en los debates sobre asuntos tecnocientíficos, aunque estos le conciernan (Bauer, Allum y Miller, 2007). Desde los años setenta, las instituciones han invertido enormes esfuerzos para obtener medidas fiables del nivel de conocimien- tos y de las actitudes del público hacia la ciencia, o sea, para medir el grado de alfabetización científica de la población. Como apuntan Gregory y Miller (1998), relacionar la comprensión que el público tiene de la ciencia con su actitud hacia ella es una cuestión de difícil resolución. Hay cierta unanimidad entre los estudiosos al señalar que esta noción de público es problemática. Para clarificar su significado, Felt (2000) analiza y cuestiona los aspectos más relevantes del proceso por el cual se establece la nítida demarcación entre científico y no-científico. Cuatro parecen ser los aspectos relevantes en este proceso. El primero tiene que ver con la noción de público lego. Con la especialización y diferenciación disciplinar de la ciencia, el ideal de científico generalista, ilustrado en cualesquiera materias científicas, se convierte en una entelequia $y$, por tanto, la divisoria entre experto y lego se desdibuja (Lévy-Leblond, 1992; Fehér, 1990). Cualquier científico es lego si se mueve en un contexto cognitivo distinto al de su especialidad. El segundo está relacionado con la imagen que tiene el público de la ciencia. Se ha mostrado de forma convincente que, en función de cómo enfoquen su atención, los individuos adoptan posiciones antagónicas acerca de la ciencia. Cuando se centran en la ciencia como un todo (sciencein-general), su imagen suele ser positiva; cuando se centran en problemas concretos (science-in-particular), pueden surgir dudas y rechazos (Michael, 1992). El tercer aspecto está relacionado con la representación que los medios hacen de los científicos. Muchas de nuestras más sólidas creencias sobre la ciencia derivan de la imagen idílica que tenemos del científico (LaFollette, 1990). El cuarto incumbe a la construcción de fronteras entre el discurso experto y el discurso social. El experto se considera a-ideológico, imparcial y objetivo, mientras que al social se le presupone un sesgo ideológico. Implementar mecanismos comunicativos de una sola vía es la exitosa estrategia que se esgrime para discriminar el conocimiento científico (genuino) del divulgativo (desvirtuado), y así separar el científico del no-científico (Hilgartner, 1990).

Sin embargo, el modelo contextual reconoce que en realidad los individuos no reciben información como si fueran contenedores vacíos, sino que la procesan activamente de acuerdo con esquemas psicosociales modelados por sus experiencias previas, sus circunstancias personales y el contexto cultural en el que se desarrollan. En las sociedades industrializadas la ciencia y la tecnología son ubicuas, lo que genera expectativas y demandas, de tal manera que es imposible vivir sin usar conocimientos científicos. El público no necesariamente tiene un genuino interés en ser instruido, 
sino que más bien tiene un interés pragmático por aquellos resultados científicos directamente relacionados con sus necesidades cotidianas. Como señalan Brossard y Lewenstein (2010), la teoría del aprendizaje muestra que la gente aprende mejor cuando los hechos y teorías tienen significado para sus vidas personales. Por ejemplo, en ciertas comunidades donde la calidad del agua es un problema, las personas, incluso las más limitadas educativamente, pueden llegar a comprender rápidamente información técnica muy compleja. Es importante señalar que el modelo contextual también reconoce la influencia que tienen las representaciones mediáticas en amortiguar o amplificar preocupaciones latentes en el público, así como la capacidad que tiene este para movilizarse y organizar grupos de presión, gracias a las redes sociales.

Desde hace años muchos organismos públicos de países democráticos han elaborado informes y recomendaciones en los que se exhorta a la promoción del diálogo entre ciencia y sociedad. Los expertos en CPCT deben prestar mayor atención a estas iniciativas institucionales para dilucidar si responden a una concepción genuina del público como verdadero actor social, con capacidad reflexiva, crítica y deliberativa para comprometerse con la ciencia, pero también para enjuiciarla y participar en la toma de decisiones, o, por el contrario, bajo esa pátina dialéctica se encubre un refinamiento más que un reemplazamiento del modelo de difusión.

En resumen, la percepción que del público tienen aquellos que comunican la ciencia o teorizan sobre ella condiciona el modo de comunicar y explotar determinado mapa conceptual. Así, por ejemplo, quien asume que el público es un recipiente vacío presto a ser llenado para que reconozca la autoridad de la ciencia, será más proclive a emplear conceptos como dise- minación del conocimiento, déficit cognitivo o cultura científica. Sin embargo, quien acepta que el público accede de manera discrecional y selectiva a la información científica y que, por tanto, es un agente interpretativo, utilizará expresiones como modelo contextual, conocimiento local o democracia deliberativa.

\section{CONCLUSIONES}

La historia conceptual de la CPCT ha pasado por varias etapas. En una primera etapa (1960-mediados de la década de 1980), dominada por la noción de alfabetización científica, se considera que el público es deficitario en conocimientos. Tanto practicantes como teóricos implementan estrategias comunicativas para reforzar la educación formal de la ciudadanía. Impera el modelo PAST y su terminología pedagógica asociada. En una segunda etapa (1985-finales de la década de 1990), lo que preocupa ya no es tanto la carencia de conocimientos (que también) como la actitud del público hacia la ciencia y, por tanto, los esfuerzos se dirigen a comprometerlo con la ciencia. Se combinan recursos didácticos con técnicas propias de las relaciones públicas. Como en la etapa anterior, el modelo PAST sigue vigente y vigoroso, aunque comienza a hablarse de la necesidad de establecer cauces de diálogo entre ciencia y sociedad. Por último, en una tercera etapa (finales de la década de 1990-actualidad), los teóricos de la CPCT reformulan el problema al plantearse que el déficit ya no recae tanto en el público como en el experto. Se insta a redefinir el relevante papel del experto y solventar, además, la supuesta crisis de legitimidad social que sufre la ciencia. Las estrategias puestas en juego intentan estimular y mejorar la participación del ciudadano en el devenir de la ciencia. A pesar de que el modelo PAST pervive y sigue pujante, son los modelos PEST y CUSP los que irrumpen con fuerza en este periodo.

\section{NOTAS}

1 En el ámbito anglosajón se habla de Public Communication of Science and Technology (PCST) o, simplemente, Science Communication, aunque esta última expresión también puede incluir la comunicación inter pares, es decir, la que se genera dentro de las comunidades científicas.
2 En el 70 Programa Marco de la UE se defiende el término ciencia en sociedad; sin embargo, en el vigente programa, Horizonte 2020, se habla de ciencia con y para la sociedad, una nueva expresión que subraya el carácter de servicio público que debe tener la ciencia en la sociedad.
3 Uno de los hitos fundamentales del movimiento PUS es el Informe Bodmer, elaborado en 1985 por la Royal Society. En él se insta a científicos y comunicadores a que hagan esfuerzos por educar al público para mejorar su comprensión de la ciencia, y así fortalecer la legitimidad social de esta. 


\section{BIBLIOGRAFÍA}

Alcíbar, M. (2007). Comunicar la ciencia. La clonación como debate periodístico. Madrid: Consejo Superior de Investigaciones Científicas.

Bauer, M. W. (1998). The medicalization of science news - from the "rocket-scalpel" to the 'gene-meteorite' complex. Social Science Information, 37, 4, pp. 731-751. Doi: 10.1177/053901898037004009.

Bauer, M. W., Allum, N. y Miller, S. (2007). What can we learn from 25 years of PUS survey research? Liberating and expanding the agenda. Public Understanding of Science, 16, 1, pp. 7995. Doi: 10.1177/0963662506071287.

Bauer, M. W. y Gregory, J. (2008). From journalism to corporate communication in post-war Britain. En Bauer, M. W. y Bucchi, M. (eds.). Journalism, Science and Society. Science Communication between News and Public Relations. London: Routledge, pp. 33-51.

Blanco, R. J. e Iranzo, J. M. (2000). Ambivalencia e incertidumbre en las relaciones entre ciencia y sociedad. Papers de Sociología, 61, pp. 89-112.

Bodmer, W. (1985). The Public Understanding of Science. London: The Royal Society.

Broks, P. (2006). Understanding Popular Science. New York: Open University Press.

Brossard, D. y Lewenstein, B. V. (2010). A Critical Appraisal of Models of Public Understanding of Science: Using Practice to Inform Theory. En Kahlor, L. y Stout, P. A. (eds.). Communicating Science. New Agendas in Communication. New York and London: Routledge, pp. 11-39.

Bucchi, M. (1998). Science and the media. Alternative routes in scientific communication. London and New York: Routledge.

Bucchi, M. (2013). Style in science communication. Public Understanding of Science, 22, 8, pp. 904-915. Doi: $10.1177 / 0963662513498202$.

Burns, T., O' Connor, J. y Stocklmayer, S. (2003). Science communication: a contemporary definition. Public Understanding of Science, 12, 2, pp. 183-202. Doi: $10.1177 / 09636625030122004$.

Bush, V. (1945). Science: the Endless Frontier. Washington: United States Government Printing.

Collins, H. y Pinch, T. (1996). El golem. Lo que todos deberíamos saber acerca de la ciencia. Barcelona: Crítica.
Durant, J. (1999). Participatory technology assessment and the democratic model of the public understanding of science. Science and Public Policy, 26, 5, pp. 313-319. Doi: 10.3152/147154399781782329.

Durant, J. (2010). Public Understanding of Science. En Hornig Priest, S. (ed.). Encyclopedia of Science and Technology Communication. Thousand Oaks: Sage, pp. 616-619.

Durant, J., Evans, G. A. y Thomas, G. P. (1989). The public understanding of science. Nature, 340, 6228, pp. 11-14. Doi: 10.1038/340011a0.

Echeverría, J. (2003). La revolución tecnocientífica. Madrid: Fondo de Cultura Económica.

Elías, C. (2008). Fundamentos de periodismo científico y divulgación mediática. Madrid: Alianza Editorial.

Elliott, R. (2012). The Medialization of Regenerative Medicine: Frames and Metaphors in UK News Stories. En Rödder, S., Franzen, M. y Weingart, P. (eds.). The Sciences' Media Connection -Public Communication and its Repercussions. Dordrecht and New York: Springer, pp. 87-105. Doi: 10.1007/978-94-0072085-5_5

European Commission (2009). Challenging Futures of Science in Society. Emerging trends and cutting-edge issues. Report of the MASIS Expert Group setup by the European Commission [en línea]. [Disponible en http://www.masis.eu/files/ reports/Emerging_trends_in_SiS.pdf].

Evans, G. y Durant, J. (1995). The relationship between knowledge and attitudes in the public understanding of science in Britain. Public Understanding of Science, 4, 1, pp. 57-74. Doi: 10.1088/0963$6625 / 4 / 1 / 004$.

Fundación Española para la Ciencia y la Tecnología (FECYT) (2015). VII Encuesta de Percepción Social de la Ciencia. Dossier informativo [en línea]. [Disponible en: http://www.idi.mineco.gob.es/stfls/ MICINN/Prensa/NOTAS_PRENSA/2015/ Dossier_PSC_2015.pdf]

Fehér, M. (1990). Acerca del papel asignado al público por los filósofos de la ciencia. En Ordoñez, J. y Elena, A. (eds.). La ciencia y su público. Madrid: Consejo Superior de Investigaciones Científicas, pp. 421-443.
Felt, U. (2000). Why Should the Public Understand Science? A Historical Perspective on Aspects of the Public Understanding of Science. En Dierkes, M. y von Grote, C. (eds.). Between Understanding and Trust. The Public, Science and Technology. Amsterdam: Harwood Academic Publishers, pp. 7-38.

Gibbons, M. (1999). Science's new social contract with society. Nature, 402, 6761, pp. c81-c84.

González García, M. I., López Cerezo, J. A. y Luján López, J. L. (1996). Ciencia, tecnología y sociedad. Una introducción al estudio social de la ciencia y la tecnología. Madrid: Tecnos.

Gregory, J. y Miller, S. (1998). Science in public. Communication, culture, and credibility. New York: Plenum Trade.

Gross, A. G. (1994). The roles of rhetoric in the public understanding of science. Public Understanding of Science, 3, 1, pp. 3-23. Doi: 10.1088/0963-6625/3/1/001.

Grundmann, R. y Cavaillé, J.-P. (2000). Simplicity in Science and its Publics. Science as Culture, 9, 3, pp. 353-389. Doi: 10.1080/713695251.

Hansen, A. (2009). Science, communication and media. En Holliman, R., Whitelegg, E. S., Smidt, S. y Thomas, J. (eds.), Investigating Science Communication in the Information Age. Implications for public engagement and popular media. New York: Oxford University Press, pp. 105-127.

Hilgartner, S. (1990). The Dominant View of Popularization: Conceptual Problems, Political Uses. Social Studies of Science, 20, 3, pp. 519-539. Doi: 10.1177/030631290020003006.

Horst, M. (2008). In search of dialogue: staging science communication in consensus conferences. En Cheng, D., Claessens, M., Gascoigne, T., Metcalfe, J., Schiele, B. y Shi, S. (eds.). Communicating Science in Social Contexts. New models, new practices. Brussels: Springer, pp. 259-274.

Horst, M. y Michael, M. (2011). On the Shoulders of Idiots: Re-thinking Science Communication as 'Event'. Science as Culture, 20, 3, pp. 283-306. Doi: 10.1080/09505431.2010.524199.

House of Lords. Select Committee on Science and Technology (2000). Science and Society; Third Report of the Session 19992000. London: HM Stationery Office. 
LaFollette, M. C. (1990). Making science our own. Public images of science, 1910-1955. Chicago: University of Chicago Press.

Lévy-Leblond, J.-M. (1992). About misunderstandings about misunderstandings. Public Understanding of Science, 1, 1, pp. 1722. Doi: 10.1088/0963-6625/1/1/004.

Lewenstein, B. (1992). The meaning of 'public understanding of science' in the United States after World War II. Public Understanding of Science, 1, 1, pp. 4568. Doi: 10.1088/0963-6625/1/1/009.

Logan, R. A. (2001). Science Mass Communication: Its Conceptual History. Science Communication, 23, 2, pp. 135-163. Doi: $10.1177 / 1075547001023002004$

Luzón, M. J. (2013). Public Communication of Science in Blogs: Recontextualizing Scientific Discourse for a Diversified Audience. Written Communication, 30, 4, pp. 428-457. Doi: 10.1177/0741088313493610.

Meyer, G. (2006). Journalism and Science: How to Erode the Idea of Knowledge. Journal of Agricultural and Environmental Ethics, 19, 3, pp. 239-252. Doi: 10.1007/s10806-005-6163-1.

Michael, M. (1992). Lay Discourses of Science: Science-in-General, Science-inParticular, and Self. Science, Technology \& Human Values, 17, 3, pp. 313-333. Doi: $10.1177 / 016224399201700303$.

Miller, J. D. (2004). Public Understanding of, and Attitudes toward, Scientific Research: What We Know and What We Need to Know. Public Understanding of Science, 13, 3, pp. 273-294. Doi: 10.1177/0963662504044908.

Miller, S. (2001). Public understanding of science at the crossroads. Public Understanding of Science, 10, 1, pp. 115-120. Doi: 10.1088/0963-6625/10/1/308.
Myers, G. (2003). Discourse Studies of Scientific Popularization: Questioning the Boundaries. Discourse Studies, 5, 2, pp. 265279. Doi: $10.1177 / 1461445603005002006$.

Nelkin, D. (1990). La ciencia en el escaparate. Madrid: Fundesco.

Nerlich, B., Dingwall, R. y Clarke, D. D. (2002). The book of life: How the completion of the Human Genome Project was revealed to the public. Health, 6, 4, pp. 445-469. Doi: 10.1177/136345930200600403.

Nisbet, M. C. (2010). Framing Science. A New Paradigm in Public Engagement. En Kahlor, L. y Stout, P. A. (eds.). Communicating Science. New Agendas in Communication. New York \& London: Routledge, pp. 40-67.

Peters, H. P. (2012). Scientific Sources and the Mass Media: Forms and Consequences of Medialization. En Rödder, S., Franzen, M. y Weingart, P. (eds.). The Sciences' Media Connection -Public Communication and its Repercussions. Dordrecht and New York: Springer, pp. 217-239. Doi: 10.1007/978-94-007-2085-5 11.

Peters, H. P. (2013). Gap between science and media revisited: Scientists as public communicators. Proceedings of the National Academy of Sciences, 110, suppl. 3, pp. 14102-14109. Doi: 10.1073.

Radder, H. (2010). The Commodification of Academic Research. Pittsburgh, PA: University of Pittsburgh Press.

Rödder, S. (2009). Reassessing the concept of a medialization of science: A story from the 'book of life'. Public Understanding of Science, 18, 4, pp. 452-463. Doi: $10.1177 / 0963662507081168$.

Sørensen, K. H., Aune, M. y Hatling, M. (2000). Against Linearity $-O n$ the
Cultural Appropriation Of Science And Technology. En Dierkes, M. y von Grote, C. (eds.). Between Understanding and Trust. The Public, Science and Technology. Amsterdam: Harwood Academic Publishers.

Tinker P. S. (2013). Communicating popular science. From deficit to democracy. New York: Palgrave Macmillan.

Trench, B. (2008). Towards an Analytical Framework of Science Communication Models. En Cheng, D., Claessens, M., Gascoigne, T., Metcalfe, J., Schiele, B. y Shi, S. (eds.). Communicating Science in Social Contexts. New models, new practices. Brussels: Springer, pp. 119-135.

Trench, B. y Bucchi, M. (2010). Science communication and emerging discipline. Journal of Science Communication, 9, 3, pp. 1-5.

Väliverronen, E. (1993). Science and the Media: Changing Relations. Science Studies, 6, 2, pp. 23-34.

Weigold, M. F. (2001). Communicating Science: A Review of the Literature. Science Communication, 23, 2, pp. 164-193. Doi: $10.1177 / 1075547001023002005$.

Weingart, P. (1998). Science and the media. Research Policy, 27, 8, pp. 869-879. Doi: 10.1016/S0048-7333(98)00096-1.

Woolgar, S. (1991). Ciencia: abriendo la caja negra. Barcelona: Anthropos.

Wynne, B. (1995). The public understanding of science. En Jasanoff, S., Markle, G., Peterson, J. y Pinch, T. (eds.). Handbook of Science and Technology Studies. Thousand Oaks, CA: Sage, pp. 361-388. 